\title{
Parameters of kinaesthesis during gaits derived from an ultrasound-based measuring system
}

\author{
R. M. Kiss \\ Department of Structures, \\ Budapest University of Technology and Economics, Hungary
}

\begin{abstract}
In order to analyze kinaesthesis of motion, the author used a ZEBRIS ultrasoundbased measuring system with single active markers. In essence, the measurement involves the determination of the spatial positions of different anatomical points on the human body while a person is stepping on firm and non-firm plates, which also have active markers attached. This type of measurement can model the correction ability of the person being examined. The provocation test, when the plate is suddenly swivelled out with a provocation unit, can model the equilibration ability of the person being investigated. In this study the kinematic and oscillation (swing) characteristics of 50 healthy persons were identified during stepping and provocation. The kinematic characteristics of stepping were identified by measuring the range of knee motion in all three directions of space and the range of motion of the shoulder girdle and pelvis in a local coordinate system of segments (shoulder girdle and pelvis). The oscillation (swing) characteristics of provocation were identified by average logarithmic decrement, Lehr's damping ratio, frequency and own frequency. Kinaesthesis can be described by analyzing the results of the measurements performed.
\end{abstract}

Keywords: ultrasound-based measuring system, kinaesthesis, proprioception, swing parameters.

\section{Introduction}

Kinaesthesis (dynamic perception) is the perception of the relationships of the moving parts of the body to each other. When examining kinaesthesis, we analyze the reiterative accuracy of well-known movements. This method is suitable for examining patients with infantile cerebral paresis and degenerative 
clinical patterns. As regards the investigation of kinaesthesis, gait analysis is very widely used. The standard deviations of different kinematic parameters (temporal, spatial, angular parameters) can model the reiterative accuracy of motion [1], which is equivalent to modelling kinaesthesis. However, the gait analysis cannot be used a short time after the injury or after the reconstruction. Kinematic parameters are not suitable for modelling the equilibration ability of the person, which means modelling the oscillation of motion after a sudden change in motion.

The ZEBRIS ultrasound-based motion analyzer system (ZEBRIS Medizintechnik GmbH, Isny, Germany), using individual markers, a measurement control program and a biomechanical model, is suitable for determining the positions of individual markers attached to different anatomical points on the human body while the person is stepping on firm (stable) ground as well as on non-firm (unstable) ground. By using a non-firm plate, the oscillation of motion can be determined after a provocation, when the plate is suddenly swivelled out with a provocation unit. The accuracy of the measuring method enables us to calculate the different kinematic as well as the oscillation (swing) parameters. The measuring method is suitable for analyzing correction and equilibration abilities, and it may be used shortly after reconstruction surgery.

This research was aimed at jointly producing a test method and corresponding biomechanical parameters suitable for modelling the kinaesthesis of motion. A further objective was to generate a databank of parameters from healthy young subjects as a basis for further investigations.

\section{Materials and methods}

\subsection{Subjects}

The positions of the anatomical points were determined for 50 healthy subjects using the method described below. Only people without any clinical history of lower extremity or shoulder joint disease or injury were involved in the study. There were 25 males (average age $22.1 \pm 5.1$ years, average height $171.9 \pm 4.9$

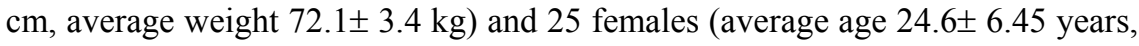
average height $168.9 \pm 7.3 \mathrm{~cm}$, average weight $63.1 \pm 7.5 \mathrm{~kg}$ ). The tests were authorized by the Science and Research Ethics Committee of Semmelweis University. Each voluntary subject provided informed written consent to perform the tests in advance.

\subsection{Measurement method, biomechanical model}

The spatial coordinates of certain anatomical points during motion were measured by a ZEBRIS CMS10 computer-controlled, ultrasound-based motion analysis system located in the Biomechanical Laboratory of the Department of Orthopaedics of Semmelweis University.

The active, individual markers attached to the anatomical points are transmitters and emit ultrasound signals at specific intervals, which are recorded 
by three markers (microphones) built in the measuring head (the measurement frequency being $100 \mathrm{~Hz}$ ). Knowing the speed of the ultrasound waves, the distances between each active marker and the microphones on the measuring head can be calculated from the time delays of the transmission. From the distances between the three microphones of the measuring head and each of the active markers and knowing the spatial coordinates of the three microphones on the measuring head, the spatial coordinates of the active markers at each moment of time during the measurement can be calculated using the method of triangulation [2] (Figure 1).

The main criteria of the measurement procedure are standardization and reproducibility. Therefore, the locations on the body of the active sensors that emit the ultrasound signals must be selected so that the possibility of displacement during measurement is excluded; the anatomical points must be properly determinable and palpable through the skin. The active markers are fixed in place, using two-sided plaster tape, to the tuberositas tibiae in order to test the motion of the lower limb and to the spina iliaca anterior superior and acromion scapulae in the shoulder girdle to test the motion of the upper body. These anatomical points are particularly useful because there is relatively little motion of the skin over osseous anatomical points during gait and other types of motion.

An important element of the examination of kinaesthesis (dynamic perception) is modelling the correction ability on non-firm (unstable) ground. The most suitable device for this is the ZEBRIS Posturomed ${ }^{\circledR}$ plate, secured by eight springs with the same spring strength in each direction (Figure 1). The Posturomed $^{\circledR}$ plate can be used as a firm plate (so that the springs do not allow motion of the plate in any direction) or as a non-firm plate (so that the springs allow motion of the plate in both horizontal directions).

Using the Posturomed ${ }^{\circledR}$ plate the oscillation of motion can be modelled with different oscillation parameters (frequency, damping parameters, etc.) after a provocation. During the provocation the plate is suddenly swivelled out with a provocation unit (Figure 1), and the tension on the springs is released when they are in an extreme position. The oscillation (swing) parameters can be calculated from the positions of the markers attached to the plate as a function of time.

\subsection{Test and assessment parameters for characterizing the kinaesthesis}

The tests are performed with males stripped to the waist and with females in bras, so that the anatomical points around the shoulder and on the lower limb are easy to access. Each of the points involved in the investigation are anatomical and anthropometrical points for the person performing the examination. Three types of tests have been set up for kinaesthesis.

\subsubsection{Test for the lower limbs: knees}

The position of tuberositas tibiae are measured during 15-20 cycles of stepping on a firm (stable) and a non-firm (unstable) plate. The ultrasound-based measuring head is located in front of the subject at 90 degrees (Figure 1). 
The range of motion of the knees is the difference between the minimum and maximum values of the spatial coordinates of the anatomical point of tuberositas tibiae to be determined during one period, in the spatial directions $x, y$ and $z$, which means in the lateral, anterior-posterior and up-down directions, respectively.

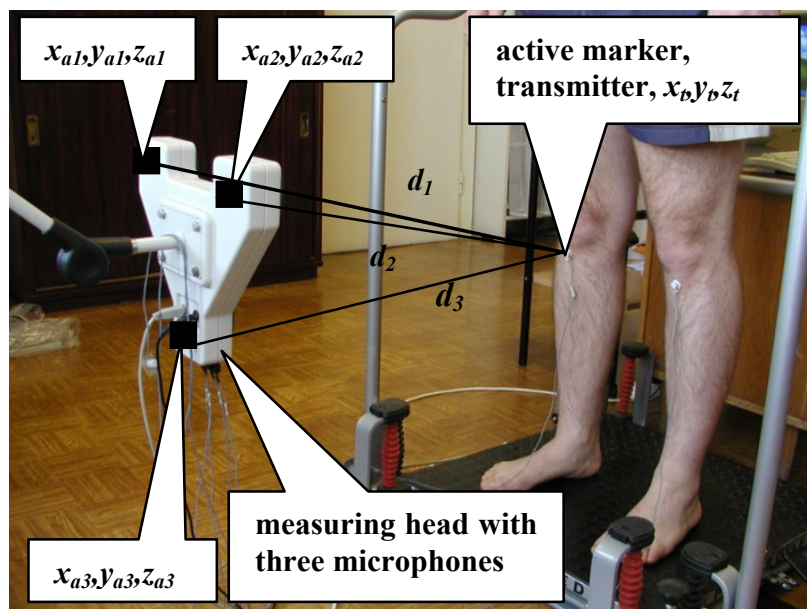

Figure 1: Calculated position of the active marker (transmitter) $\left(x_{t}, y_{t}, z_{t}\right)$ from the positions of the three microphones of the measuring head $\left(x_{a i}, y_{a i}, z_{a i}\right)$, and from the distances between the three microphones and an active marker $\left(d_{i}\right)$ by the triangulation method. The picture shows the measurement arrangement during the test for the lower limbs.

\subsubsection{Test for the upper body: shoulder girdle and pelvis}

The position of acromions and ASIS are measured during 15-20 cycles of stepping on a firm (stable) and a non-firm (unstable) plate. The ultrasound-based measuring head is located in front of the subject at 90 degrees (Figure 2a).

Before starting the measurement, in the calibration phase, the spatial position of the left and right acromion must be recorded with the subject in a natural posture. Using the positions of the acromions the local coordinate system of the shoulder girdle can be determined, as the axis $\xi$ of the local coordinate system of the shoulder girdle is the line connecting the left and right acromions. Axis $\eta$ of the local coordinate system is a line perpendicular to axis $\xi$ at the midpoint of the line connecting the left and right acromions. Axis $\zeta$ of the local coordinate system is a line perpendicular to the plane defined by axes $\xi$ and $\eta$ at the intersection of the two axes (Figure 3a). Flexion-extension of the shoulder girdle $(\alpha)$, which occurs upon rotation of the line connecting the left and right acromions around the axis $\xi$ of the local coordinate system, that is, in the $\eta-\zeta$ plane, corresponds to the angle enclosed by the line connecting the left and right acromions and the axis $\eta$ (Figure 3a) during motion. The tilt of the shoulder 
girdle $(\beta)$, which changes with the rotation of the line connecting the left and right acromions around the axis $\eta$ of the local coordinate system, that is, in the $\xi-\zeta$ plane, corresponds to the angle enclosed by the line connecting the left and right acromions and the axis $\xi$ (Figure 3a) during motion. The rotation angle of the shoulder girdle $(\gamma)$, which changes upon rotation of the line connecting the left and right acromions around the axis $\zeta$ of the local coordinate system, that is, in the $\xi-\eta$ plane, corresponds to the angle enclosed by the line connecting the left and right acromions and the axis $\xi$ (Figure 3a) during motion. The ranges of the different motions of the shoulder girdle correspond to the differences between the minimum and maximum values of the angles (flexion-extension, tilt, rotation) of the shoulder girdle that are determined during one period.

The local coordinate system of the pelvis can be determined - similarly to the local coordinate system of the shoulder girdle - before starting the measurement, from the spatial positions of the anatomical points of the left and right spina iliaca anterior superior (Figure $3 b$ ). The range of flexion-extension of the pelvis $(\delta)$, the tilt of the pelvis $(\phi)$, the rotation angle of the pelvis $(\rho)$ is the difference between the minimum and maximum values of the angles that are determined during one period.

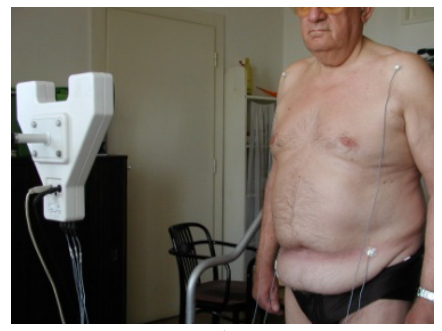

a)

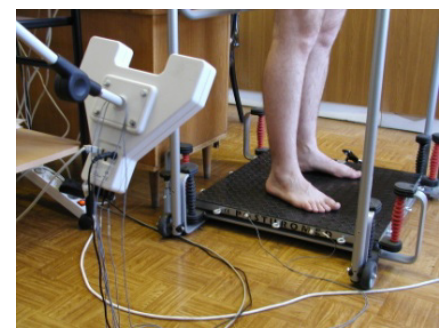

b)

Figure 2: Measurement arrangement a) during the test for the upper body (test for shoulder girdle and pelvis) and $b$ ) during the provocation.

\subsubsection{Testing for provocation}

Test for provocation includes three separate measurements, when the subject stands on both limbs or on the right or the left foot. The springs are released, and the plate suddenly moves in a horizontal direction, so that the subject has to balance and re-equilibrate as the plate moves. The spatial coordinates of the two active sensors placed on the moving plate are recorded. The ultrasound-based measuring head is located to the side of the subject at 30 degrees (Figure 2b).The lateral displacement of the measuring plate as a function of time can be calculated from the spatial coordinates of the markers fastened to the measuring plate as a function of time, where the coordinate $x$ (in the lateral direction, which is parallel to the direction of provocation) as a function of time constitutes a damping curve (Figure 4). 


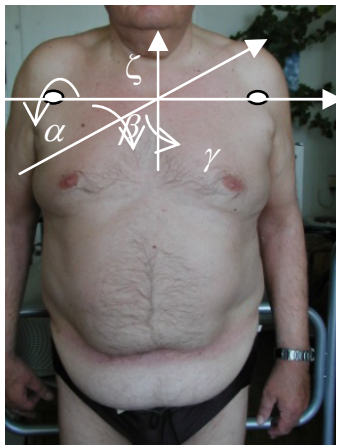

a)

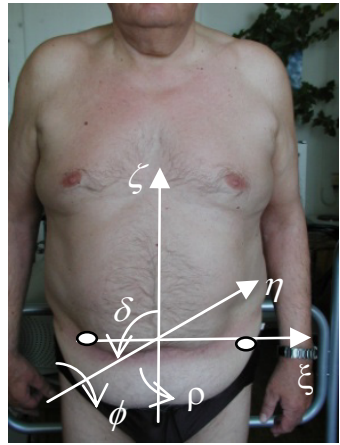

b)

Figure 3: Definition of the local coordinate system a) of the shoulder girdle and motions of the shoulder girdle and $b$ ) of the pelvis and motions of the pelvis (in the positive direction of the coordinate axis a clockwise angle will be positive).

As regards the analysis of kinaesthesis, the value of logarithmic decrement is only nearly constant as the system is not closed and there may be a number of disturbing factors. The amplitude measured at the moment $t=0$ of the test may be particularly disturbed due to the start-up of the system. It is expedient to commence the test at the moment $t_{1}$ and to determine the average value of the dynamic characteristics. The average logarithmic decrement is the average of the natural-based logarithm of the ratio of the amplitudes in an identical direction of two periods remote in time:

$$
\Lambda_{i}=\frac{1}{i-1} \ln \frac{K_{1}}{K_{i}},
$$

where $K_{1}$ is the amplitude at the moment $t=t_{1}$ (Figure 4) and $K_{i-1}$ is the amplitude at the moment $t=t_{i-1}$ (Figure 4).

The frequency $[1 / \mathrm{s}]$ is the number of repetitions in a unit of time, or the rapidity of swing:

$$
f=\frac{i-1}{t_{i}-t_{1}},
$$

where $T$ is the swing time in seconds.

Lehr's damping ratio (damping ratio, ratio $\mathrm{D}$, damping factor) is the ratio of actual and critical damping:

$$
D=\frac{\Lambda}{\sqrt{\Lambda^{2}+4 \pi^{2}}},
$$

where $\Lambda$ is the logarithmic decrement and $\pi$ is 3.14 .

Own frequency is

$$
f_{0}=\frac{1}{T \sqrt{1-D^{2}}},
$$

where $T$ is the swing time in seconds and $D$ is Lehr's damping number. 


\subsection{Statistical analysis}

Data processing and statistical analyses were performed using MS Excel based software that we developed. In the case of each subject examined, we calculated the average and the standard deviation of the biomechanical characteristics from the measurement results of the motion cycles recorded, and these data were further processed. The uniformity of the standard deviations was checked by an F-test; significance levels of the differences between the average values of identical parameters were determined by a one-sample t-test, applying a symmetrical critical range on the dominant and opposite sides.

\section{Results}

The results of the test are summarized in Tables 1-3.

\section{Discussion}

The standard deviations of different kinematic determined during gait can model kinaesthesis. However, the correction ability cannot be analyzed during gait on a fixed, stable walkway or a treadmill. Our measuring method is able to model the correction ability of the subject investigated because the motion of the subject can analyzed while a stepping on a non-firm (unstable) plate. The kinematic parameters are not suitable for modelling the equilibration ability after a sudden change in motion. During the provocation test, when the plate is suddenly swivelled out with a provocation unit in the horizontal direction, the subsequent motion of the plate is determined. The value of the coordinate $x$ (parallel with the provocation direction) as a function of time is a damping curve; from it the different oscillation (swing) parameters could be calculated.

It is known from the literature [3] that the ranges of motion of the knee during a gait cycle are $2-3 \mathrm{~cm}$ in the lateral direction and $3-5 \mathrm{~cm}$ in elevation, and these values are similar to our results for the lower extremities test when subjects were stepping on a firm plate (Table 1). By analyzing the results of the lower extremities test, it can also be established that the correction for non-firm ground can be solved with smaller anterior-posterior and up-down motions and wider right-left motions (Table 1). The standard deviation of the motion of the knee is bigger when stepping on a non-firm plate than it is when stepping on a firm plate (Table 1). From both results it can be established that the reiterative accuracy of motion on a non-firm plate is failing. The trend of these results is similar to the trend of gait parameters measured after injury $[4,5]$, in elderly people $[6,7]$ or in cases of arthritis [8,9].

The tilt and rotation of the shoulder and pelvis (Table 2), established while stepping on a firm plate, are similar to the results found in the literature $[3,6,7]$. The flexion-extension of the pelvis during stepping (Table 2) is smaller than the flexion-extension during gait $[3,6,7]$ because the flexion-extension of the pelvis plays a less important role in the forward motion of the gait. The lack of reiterative accuracy of motion on a non-firm plate establishes the greater motion of the pelvis and shoulder on a non-firm plate. 


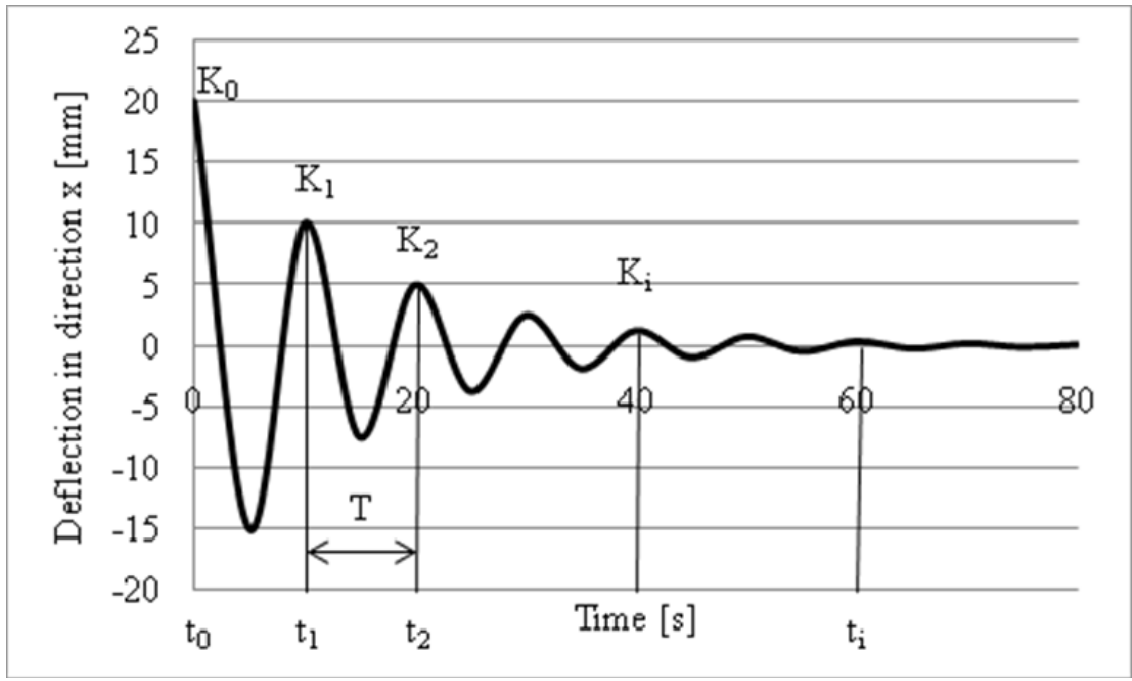

Figure 4: Horizontal $(x)$ coordinate of the active sensors attached on the plate during tests of provocation. Coordinate $x$ as a function of time yields a damping curve. Factors required for calculating the swing parameters (the amplitude $K_{i}$ at the moment $t=t_{i}, T$ is the swing time).

Table 1: $\quad$ Range of knee motion [mm] while stepping on firm and non-firm plates (average \pm standard deviation).

\begin{tabular}{|l|l|l|l|l|}
\hline Direction & \multicolumn{3}{|l|}{ Stepping on firm plate } & Stepping on non-firm plate \\
\hline & Dominant side & $\begin{array}{l}\text { Non-dominant } \\
\text { side }\end{array}$ & Dominant side & $\begin{array}{l}\text { Non-dominant } \\
\text { side }\end{array}$ \\
\hline $\begin{array}{l}x \text { (right- } \\
\text { left) }\end{array}$ & $27.63 \pm 1.82$ & $25.4 \pm 2.36$ & $42.36 \pm 4.47^{\dagger}$ & $38.62 \pm 4.42^{\dagger}$ \\
\hline $\begin{array}{l}y \quad \text { (ant- } \\
\text { post) }\end{array}$ & $191.65 \pm 9.16$ & $183.12 \pm 10.45$ & $149.98 \pm 15.51^{\dagger}$ & $142.24 \pm 19.40^{\dagger}$ \\
\hline $\begin{array}{l}z \quad \text { (up- } \\
\text { down) }\end{array}$ & $46.23 \pm 3.13$ & $44.86 \pm 8.34$ & $24.56 \pm 6.94^{\dagger}$ & $19.24 \pm 7.76^{\dagger}$ \\
\hline
\end{tabular}

† Significant difference between the average values of parameters compared to parameters while stepping on a firm plate.

The oscillation (swing parameters), as the average logarithmic decrement, Lehr's damping ratio, the frequency and the own frequency enable modelling of the equilibration ability. The subjects could damp the motion of the plate when Lehr's damping ratio was less than 1 in all three examinations (Table 3). The results show that the dominant side plays an important role in damping of the plate motion because it is significantly higher than the Lehr's damping ratio of 
Table 2: $\quad$ Range of motion of pelvis and shoulder girdle [degree] while stepping on firm and non-firm plates (average \pm standard deviation).

\begin{tabular}{|l|l|l|l|l|}
\hline Type of & \multicolumn{2}{|l|}{ Stepping on firm plate } & \multicolumn{2}{l|}{ Stepping on non-firm plate } \\
\hline motion & Shoulder & Pelvis & Shoulder & Pelvis \\
\hline $\begin{array}{l}\text { Flexion- } \\
\text { extension }\end{array}$ & $0.91 \pm 0.23$ & $1.42 \pm 0.34$ & $1.16 \pm 0.41$ & $1.67 \pm 0.53$ \\
\hline Tilt & $3.32 \pm 0.57$ & $2.66 \pm 0.46$ & $4.21 \pm 0.79^{\dagger}$ & $7.38 \pm 0.73^{\dagger}$ \\
\hline Rotation & $3.86 \pm 0.67$ & $5.58 \pm 0.58$ & $6.96 \pm 1.07^{\dagger}$ & $7.46 \pm 0.88^{\dagger}$ \\
\hline
\end{tabular}

Table 3: Oscillation (swing) characteristics during the provocation test (average \pm standard deviation).

\begin{tabular}{|l|l|l|l|l|}
\hline Standing on & \multicolumn{1}{|c|}{$\Lambda$} & \multicolumn{1}{|c|}{$D$} & \multicolumn{1}{c|}{$f[1 / s]$} & \multicolumn{1}{c|}{$f_{0}[1 / s]$} \\
\hline & 1.321 & 0.2058 & 0.003068 & 0.003132 \\
both limbs & \pm 0.044 & \pm 0.0164 & \pm 0.000344 & \pm 0.00358 \\
\hline & 1.278 & 0.1992 & 0.003116 & 0.003173 \\
dominant & \pm 0.095 & \pm 0.0248 & \pm 0.000343 & \pm 0.000348 \\
limb & & & & \\
\hline non-dominant & 0.483 & 0.0766 & 0.003181 & 0.003187 \\
limb & $\pm 0.212^{\dagger,}$ & $\pm 0.0419^{\dagger, \dagger}$ & \pm 0.000375 & \pm 0.000355 \\
\hline
\end{tabular}

$\Lambda$ - average logarithmic decrement, $D$ - Lehr's damping ratio, $f$ - frequency, $f_{0}-$ own frequency. ${ }^{\dagger}$ Significant difference between the average values of parameters compared to parameters measured while standing on both limbs. "Significant difference between the average values of parameters compared to parameters measured while standing on the dominant limb.

standing on a non-dominant limb (Table 3). From these results it can be established that the damping effect of the subject depends on the ability of the dominant side because the damping ratio when standing on both limbs is similar to that when standing on the dominant side (Table 3). On the basis of our test, the average logarithmic decrement and Lehr's damping ratio prove the dependence on lateral dominance. The frequency and the own frequency are independent of lateral dominance because there was no significant difference between standing on the dominant or on the non-dominant side (Table 3). These parameters depend on the weight of the subject and their ability to balance their whole body because there was no significant difference between the frequency and the own frequency, or between the parameters determined while standing on both limbs or on a single limb (Table 3).

The measurement method developed and the corresponding kinematic and oscillation (swing) parameters enable determination of the kinaesthesis of the motion.

The measuring method can be performed shortly after injuries, including after orthopaedic injuries. Stepping on a non-firm plate can model the correction ability of investigated persons. The provocation test and the swing parameters calculated from it can model the equilibration ability. Lehr's damping ratio and 
the own frequency are characteristic of the subject examined; they are substantially affected by various orthopaedic injuries, surgeries, age and sporting activities.

\section{Acknowledgement}

The research described in this paper was supported by a grant from the National Scientific Research Found (OTKA), Hungary, project No: T049471.

\section{References}

[1] Kirtley, C., Clinical gait analysis. Theory and practice. Elsevier Churchill Livingstone: Edinburgh, 2006.

[2] Kiss, R.M., Kocsis, L. \& Knoll, Zs. Joint kinematics and spatial temporal parameters of gait measured by an ultrasound based system. Medical Engineering \&Physics 26, pp.611-620, 2004.

[3] Winter, D.A., Biomechanics and motor control of human movement. WileyInterscience Publication: New York, 1990.

[4] Theoret, D. \& Lamontage, M., Study on three-dimensional kinematics and electromyography of ACL deficient knee participants wearing a functional knee brace during running. Knee Surgery Sports Traumatology and Arthroscopy 14, pp.555-563, 2006.

[5] Torry, M.R., Decker, M.J. \& Ellis, H.B., Mechanisms of compensating for anterior cruciate ligament deficiency during gait. Medicine and Science in Sports and Exercises 36, pp.1403-1412, 2004.

[6] Bejek, Z. Paróczai, R., Illyés, Á. \& Kiss, R.M., The influence of walking speed on gait parameters in healthy people and in patients with osteoarthritis. Knee Surgery Sports Traumatology Arthroscopy 14, pp. 612622, 2006.

[7] Riley, P.O., DellaCroce, U. \& Kerrigan, D.C., Effect of age on lower extremity joint moment contributions to gait speed. Gait and Posture 14, pp. 264-270, 2001.

[8] Al-Zahrani, K.S. \& Bakheit, A.M. A study of gait characteristic of patients with chronic osteoarthritis of the knee. Disabil Rehabil 24, pp. 275-280, 2002.

[9] Börjesson, M., Weidenhielm, L., Atsson, E. \& Olsson, E., Gait and clinical measurements in patients with knee osteoarthritis after surgery: prospective 5-year follow-up study. The Knee 12, pp.121-127, 2005. 